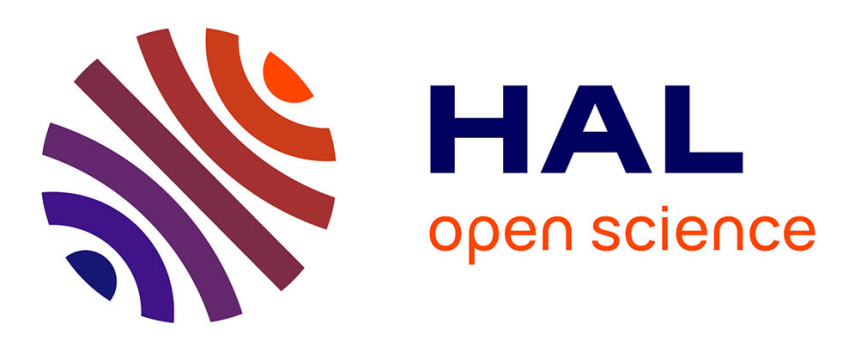

\title{
The degree measure as utility function over positions in graphs and digraphs
}

René van den Brink, Agnieszka Rusinowska

\section{To cite this version:}

René van den Brink, Agnieszka Rusinowska. The degree measure as utility function over positions in graphs and digraphs. European Journal of Operational Research, 2022, 299 (3), pp.1033-1044. 10.1016/j.ejor.2021.10.017 . hal-03513560

\section{HAL Id: hal-03513560 \\ https://hal.science/hal-03513560}

Submitted on 5 Jan 2022

HAL is a multi-disciplinary open access archive for the deposit and dissemination of scientific research documents, whether they are published or not. The documents may come from teaching and research institutions in France or abroad, or from public or private research centers.
L'archive ouverte pluridisciplinaire HAL, est destinée au dépôt et à la diffusion de documents scientifiques de niveau recherche, publiés ou non, émanant des établissements d'enseignement et de recherche français ou étrangers, des laboratoires publics ou privés. 


\title{
The degree measure as utility function over positions in graphs and digraphs ${ }^{\sqrt{2}}$
}

\author{
René van den Brink ${ }^{1}$ \\ Department of Economics, and Tinbergen Institute, VU University \\ De Boelelaan 1105, 1081 HV Amsterdam, The Netherlands \\ Agnieszka Rusinowska \\ ${ }^{a}$ CNRS - Paris School of Economics, Centre d'Economie de la Sorbonne \\ 106-112 Bd de l'Hôpital, 75647 Paris Cedex 13, France
}

\begin{abstract}
We explore the possibility to compare positions in different directed and undirected graphs. We assume an agent to have a preference relation over positions in different weighted (directed and undirected) graphs, stating pairwise comparisons between these positions. Ideally, such a preference relation can be expressed by a utility function, where positions are evaluated by their assigned 'utility'. Extending preference relations over the mixture set containing all lotteries over graph positions, we specify axioms on preferences that allow them to be represented by von Neumann-Morgenstern expected utility functions. For directed graphs, we show that the only vNM expected utility function that satisfies a certain risk neutrality, is the function that assigns to every position in a weighted directed graph the same linear combination of its outdegree and indegree. For undirected graphs, we show that the only vNM expected utility function that satisfies this risk neutrality, is the degree measure that assigns to every position in a weighted graph its degree. In this way, our results provide a utility foundation for degree centrality as a vNM expected utility function. We obtain the results following the utility approach to the Shapley value for cooperative transferable utility games of [42], noticing that undirected graphs form a subclass of cooperative games as expressed by [19]. For directed graphs, we extend this result to a class of generalized games. Using the relation between cooperative games and networks, we apply our results to some applications in Economics and Operations Research.
\end{abstract}

Keywords: group decisions and negotiations, weighted graph, degree centrality, von Neumann-Morgenstern expected utility function, cooperative game

\footnotetext{
This research has been initiated when René van den Brink was Visiting Professor at the Centre d'Economie de la Sorbonne of the University of Paris 1. Agnieszka Rusinowska acknowledges the support by the National Agency for Research (Agence Nationale de la Recherche), Project DynaMITE (ANR-13-BSH1-0010-01). Both authors acknowledge the support by the Labex OSE (ANR-10-LABX-93-01). They thank particularly Stefano Moretti for pointing out the relation between graphs and cooperative TU-games.

Email addresses: jrbrink@feweb.vu.nl (René van den Brink), agnieszka.rusinowska@univ-paris1.fr (Agnieszka Rusinowska)

${ }^{1}$ Corresponding author.
} 


\section{Introduction}

Network theory and network centrality play a growing role in the analysis of economic organizations. Network theory is developed in a wide variety of disciplines. The fact that the theory of economic and social networks have a strong connection is illustrated by, for example, the often cited book Social and Economic Networks of [30]. Whereas economic networks usually study economic processes that take place on a network (such as trade networks, firm hierarchies, etc.), in social networks usually the analysis of centrality is solely based on the information about the network structure. Although social network centrality measures are often applied in economic models, a foundation of network centrality measures as utility functions is missing. The main goal of the underlying paper is to provide a first step in such a foundation.

In this paper, we consider weighted (directed and undirected) networks, where each bilateral link has a nonnegative weight. We assume an agent to have a preference relation over network positions in different weighted directed networks, stating pairwise comparisons between these network positions. Under certain conditions, this preference relation can be expressed by a utility function over network positions, meaning that the agent prefers a particular network position over another position if and only if the 'utility' of the first position is greater than that of the second position. Extending the preference relation over the mixture set containing all lotteries over network positions, we specify axioms on the preferences that allow them to be represented by a von Neumann-Morgenstern expected utility function ([61]). In this way, we can compare different network positions in different networks. Questions that can be addressed in this context are, for example, does an agent prefer to be the top of a small organization or a middle manager in a large organization (directed networks), or does an agent prefer to be in the fringe of a large network or in the center of a small network (undirected networks)?

Mathematically, a utility function over network positions as described above, is identical to a social network power measure (for directed networks) or centrality measure (for undirected networks). The study of network centrality originates from the social network literature where different types of network centrality are distinguished such as degree, closeness, betweenness, prestige, etc. Various centrality measures are developed measuring these types of centrality. In the literature on cooperative transferable utility (TU) games, [42] developed a utility theory where an agent has a preference relation over different player roles in different cooperative TU-games. Requiring certain axioms on these preference relations, he gave interpretations to game solutions, such as the Shapley value ([49]) and Banzhaf value ([2]) as von Neumann-Morgenstern expected utility functions over player roles in different games. A crucial 
axiom is ordinary risk neutrality which involves the uncertainty that arises from lotteries.

Applied to networks, neutrality to ordinary risk means that an agent is indifferent between taking a position in a convex combination of two networks, and playing a lottery over the two networks with the corresponding probabilities. Assuming some regularity axioms on network preference relations, we show that regular preferences are neutral to ordinary risk if and only if they can be represented by a utility function that assigns to every network position in any network a linear combination of its outdegree and indegree (for directed networks), respectively, its degree (for undirected networks) 2 In this way, the degree measures can be seen as a von Neumann-Morgenstern expected utility function for positions in networks.

For undirected networks, this also gives a third important interpretation of the degree measure, besides its known myopic and farsighted interpretation. The myopic interpretation of the degree measure is that it is a centrality measure that only takes account of direct relations, and completely ignores indirect relations. However, it can also be considered as a farsighted centrality measure in the sense that for a simple connected network, the probability that a random walk will be found at a node in the limit of long time is proportional to the degree of that node, see e.g. [36] page 159.

We finally apply our results to three applications in Economics and Operations Research. As argued by [19], weighted undirected graphs can be equivalently represented by cooperative 2-additive games, being zero-normalized games where the worth of every coalition containing at least two players equals the sum of the worths of its two-player subcoalitions (see Appendix A for more details). This implies that all applications in Economics and Operations Research that are modelled as 2-additive games can be represented as graphs. Using this insight, we apply our results to three classes of 2-additive games: broadcasting games, queueing games and telecommunication games.

Related literature. In this paper, we use the approach of [42] to interpret the degree centrality measure as an expected utility function. Building on the utility theory over mixture sets of [29], [42] develops a foundation of the Shapley value as a von Neumann-Morgenstern expected utility function reflecting preferences over player positions in cooperative games which permits to compare being different players in different games, see also [41, 43, 44], for related studies on this issue. [42] extends the preference relation to lotteries over games and shows that the Shapley value is an expected utility function reflecting preferences neutral to both ordinary and strategic risk ${ }^{3}[19]$ argues that any undirected network can be represented by a TU-game, more specifically by a so-called 2-additive game, and shows that the Shapley

\footnotetext{
${ }^{2}$ Under these condition, also any positive affine transformation of the degree measure represents these preferences.

${ }^{3}$ For an overview of Roth's approach to the Shapley value, see also [40].
} 
value of the associated game assigns to every player half of its degree in the network, see Appendix A. Whereas [42] uses both ordinary and strategic risk neutrality, we only need to consider the first type of risk.

The degree measure is one of the most natural and famous centrality concepts which can be seen as an index of the node's communication ability. A simple network is a network where the links have either weight zero or one, reflecting that we only know whether a link is present or not, but nothing is known about the 'strength' of the links. In such simple networks, the degree of a node boils down to the number of links formed by that node, i.e. the number of its neighbours. Inspired by some experimental studies, [50] presents the degree centrality as a measure to be used for predicting the behavior of individuals in small groups. [37] and [55] analyze the degree centrality from an axiomatic point of view.

The present paper is also related to the literature on social networks and centrality; for some pioneering articles see e.g. [3, 4], [31], [5], [46], [22, 23], and [8, 9], for surveys see e.g. [10], [26], [30], and [36]. Our research is particularly related to works using the axiomatic approach to centrality measures. This stream of literature focusses mainly on specific centrality measures. For instance, [24] characterizes axiomatically the degree, decay and closeness centralities. [12] and [55] axiomatize power measures for directed networks. Some prestige and eigenvector-related centrality measures are characterized in [39], [51], [20], and [32]. [7] characterizes the standard centrality measures within a unified framework and shows that they all are characterized by a common set of axioms. Although the present paper also uses the axiomatic approach to centrality measures and characterizes the degree measure, our main aim is to show that this measure can be interpreted as an expected utility function reflecting preferences over positions in weighted networks.

An issue closely related to centrality is the ranking of nodes which is treated by using a ranking method. Formally, a ranking method assigns to every (weighted) network a (complete) preorder on the set of nodes. This preorder is a ranking of the nodes in order of 'importance' or 'centrality' in the network. Various ranking methods are characterized in the literature, in particular, methods based on directed networks, see e.g. [45] for the ranking by outdegree on the class of tournaments, see also [28] and [11] for the ranking by Copeland score ([15]), [13], [56] for the ranking by outdegree for arbitrary directed networks, [59] for the degree ratio ranking method for directed networks, and [57] for the outflow ranking method for weighted directed networks. [21] investigates ranking of items in a network determined by choice of utility function. More precisely, the network is transformed into an exchange economy and the competitive equilibrium prices of the network nodes are used for the ranking. Some recent studies characterize ranking methods based on evaluations or citations which consider one-sided settings (e.g. [17]) and ranking methods in two-sided settings (e.g. [18]). An important difference 
between such ranking methods and the topic of this paper is that ranking methods only compare the positions in one and the same network. This is useful if one wants to rank, for example, teams in a sports competition, alternatives in a preference relation, web pages on the internet, etc. Besides such comparisons within one network, a main goal of the underlying paper is to compare positions in different networks. For example, we want to know if an agent prefers a 'central' position in a small network to a position in the fringe of a large network. In order to answer these questions we need to be able to compare positions in different networks.

This paper is organized as follows. In Section 2, we discuss preliminaries on networks and [29]'s expected utility theory over mixture sets. In Section 3 , we characterize linear combinations of the out- and indegree measures as von Neumann-Morgenstern expected utility functions for directed networks. We use as intermediary results axiomatizations of these measures as power measure for directed networks, and relate properties of network power measures to properties of preference relations over directed network positions. In Section 4, we consider undirected networks, which form a special subclass of directed networks. In Section 5 we discuss three applications. Section 6 contains concluding remarks. The paper ends with three appendices: Appendix A on networks as cooperative games, Appendix B containing proofs, and Appendix $\mathrm{C}$ showing logical independence of the axioms in our main theorem.

\section{Preliminaries}

In this section, we present basic concepts and notation that will be used in the paper.

Weighted directed graphs. A weighted directed graph, or weighted digraph for short, is a pair $(N, \omega)$ consisting of a finite set of nodes $N \subset \mathbf{N}$ that can represent individuals or agents, and a directed weight function $\omega: N \times N \rightarrow \mathbb{R}_{+}$assigning to every $\operatorname{arc}(i, j) \in N \times N$ (i.e., to every ordered pair of nodes) a nonnegative weight $\omega(i, j)$. An arc $(i, j)$ represents a directed bilateral relationship between nodes $i$ and $j$. In this model, a weight $\omega(i, j)=0$ means that there is no arc from node $i$ to node $j$. We only consider weighted digraphs that satisfy $\omega(i, i)=0$ for every $i \in N$. We denote the collection of all directed weight functions on $N$ by $\mathcal{W} \mathcal{D}^{N}$. We often refer to a weighted digraph simply as a directed graph or digraph. Since we take the set of nodes $N$ to be fixed, we represent a weighted digraph $(N, \omega)$ by its directed weight function $\omega$.

A power measure for digraphs is a function $f: \mathcal{W} \mathcal{D}^{N} \rightarrow \mathbf{R}^{N}$ that assigns a real number to every node in every digraph. The outdegree of node $i$ in weighted digraph $\omega$ is the sum of the weights of $i$ 's 
outgoing arcs, and thus is defined by

$$
\operatorname{out}_{i}(\omega)=\sum_{j \in N} \omega(i, j)
$$

The indegree of node $i$ in weighted digraph $\omega$ is the sum of the weights of its ingoing arcs, and is given by

$$
i n_{i}(\omega)=\sum_{j \in N} \omega(j, i)
$$

The outdegree-, respectively indegree, measure is the centrality measure that assigns to any node $i$ in any digraph $\omega$ its outdegree $\operatorname{out}_{i}(\omega)$, respectively its indegree $i_{i}(\omega)$. Let $\Pi(N)$ be the collection of all permutations $\pi: N \rightarrow N \sqrt[4]{4}$ For a digraph $\omega \in \mathcal{W D}^{N}$ and a permutation $\pi \in \Pi(N)$, the permuted digraph $\pi \omega \in \mathcal{W} \mathcal{D}^{N}$ is given by $\pi \omega(i, j)=\omega\left(\pi^{-1}(i), \pi^{-1}(j)\right)$ for every $(i, j) \in N \times N$.

We say that node $i \in N$ is isolated in $\omega \in \mathcal{W D}^{N}$ if $\omega(i, j)=\omega(j, i)=0$ for all $j \in N$. We denote the set of weighted digraphs where $i$ is an isolated node by $\mathcal{W} \mathcal{D}_{i}^{N}$. Further, we denote by $\omega^{0} \in \mathcal{W} \mathcal{D}^{N}$ the empty digraph given by $\omega^{0}(i, j)=0$ for all $i, j \in N$. Furthermore, $\omega^{i} \in \mathcal{W} \mathcal{D}^{N}$ is the standard outward oriented star graph with $i$ as center, given by $\omega^{i}(i, j)=1$ for all $j \in N \backslash\{i\}$, and $\omega^{i}(h, j)=0$ otherwise.

Weighted undirected graphs. A weighted undirected graph is a pair $(N, \omega)$ consisting of a finite set of nodes $N \subset \mathbf{N}$ and a weight function $\omega: L^{c} \rightarrow \mathbb{R}_{+}$, where $L^{c}=\{\{i, j\} \mid i, j \in N, i \neq j\}$ denotes the complete undirected graph on $N$. An element $\{i, j\} \in L^{c}$ is a subset of $N$ of size two and is called a link. A link $\{i, j\}$ represents a certain bilateral relationship between nodes $i$ and $j$. A weight function gives a nonnegative weight $\omega(\{i, j\})$ to every link that can be interpreted as the 'importance' or 'strength' of that relationship. By $\mathcal{W G}^{N}$ we denote the collection of all weight functions on $N$. When there is no confusion, in this paper we refer to a weighted undirected graph simply as a graph. A graph with $\omega(\{i, j\}) \in\{0,1\}$ for all $\{i, j\} \in L^{c}$, is usually called a simple graph. A simple graph just describes the relationships that are present, but says nothing about their intensity. Since $N$ is assumed to be fixed, we represent a graph $(N, \omega)$ by the weight function $\omega:^{5}$

A centrality measure for undirected graphs is a function $f: \mathcal{W G} \mathcal{G}^{N} \rightarrow \mathbf{R}^{N}$ that assigns a real number to every node in every undirected graph that reflects the 'centrality' of the nodes in the graph. The degree of node $i \in N$ in graph $\omega$ is defined as the sum of the weights of all links containing $i$, and

\footnotetext{
${ }^{4}$ A permutation is a bijection $\pi: N \rightarrow N$, and thus for every $i \in N$ there is exactly one $j \in N$ such that $\pi(j)=i$.

${ }^{5}$ Notice that $\omega(i, j)$, respectively, $\omega(\{i, j\})$ clarifies whether we consider directed graphs or undirected graphs. Moreover, the cases of directed and undirected graphs are presented in separate sections, i.e., in Sections 3 and 4, respectively, and therefore it will be clear from the context if we deal with $\mathcal{W} \mathcal{D}^{N}$ or $\mathcal{W G}^{N}$.
} 
thus is given by

$$
d_{i}(\omega)=\sum_{j \in N \backslash\{i\}} \omega(\{i, j\})
$$

The degree measure is the centrality measure that assigns to any node $i$ in any graph $\omega$ its degree $d_{i}(\omega)$.

We can consider the class of undirected graphs as a special subclass of directed graphs, where an undirected graph $\omega \in \mathcal{W G}^{N}$ can be represented by the digraph $\bar{\omega} \in \mathcal{W D}^{N}$ given by $\bar{\omega}(i, j)=\bar{\omega}(j, i)=$ $\omega(\{i, j\})$ for all $\{i, j\} \in L^{c}$. Note that, in that case, both the indegree as well as the outdegree of every node $i \in N$ in $\bar{\omega}$, equals $i$ 's degree in $\omega$, showing that both the outdegree as well as the indegree can be seen as generalizations of the degree.

For a graph $\omega \in \mathcal{W} \mathcal{G}^{N}$ and a permutation $\pi \in \Pi(N)$, the permuted graph $\pi \omega \in \mathcal{W} \mathcal{G}^{N}$ is given by $\pi \omega(\{i, j\})=\omega\left(\left\{\pi^{-1}(i), \pi^{-1}(j)\right\}\right)$ for every $\{i, j\} \in L^{c}$.

We say that node $i \in N$ is isolated in $\omega \in \mathcal{W G}^{N}$ if $\omega(\{i, j\})=0$ for all $j \in N$. We denote the set of weighted undirected graphs where $i$ is an isolated node by $\mathcal{W G} \mathcal{G}_{i}^{N}$. Further, we denote by $\omega^{0} \in \mathcal{W G}^{N}$ the empty graph given by $\omega^{0}(\{i, j\})=0$ for all $i, j \in N$. By $\omega^{i} \in \mathcal{W G} \mathcal{G}^{N}$, we denote the simple star graph with $i$ as center given by $\omega^{i}(\{i, j\})=1$ for all $j \in N \backslash\{i\}$ and $\omega^{i}(\{h, j\})=0$ if $i \notin\{h, j\}$.

Expected utility. We recapitulate the utility theory on mixture sets of [29] (for some related works and literature on linear utility representation theorems, see e.g. [53, 54], and [35]). Consider a set $M$. The (simple) lottery between two elements $a, b \in M$ where element $a$ occurs with probability $p \in[0,1]$ is denoted by $[p a ;(1-p) b]$. A set $M$ is a mixture set if for any $a, b \in M$ and any $p \in[0,1]$, the lottery [pa; $(1-p) b$ ] also belongs to $M$. Notice that this implies that also all compound lotteries, i.e. lotteries over lotteries, etc., belong to $M$. It is assumed that for all $a, b \in M$ and $p, q \in[0,1]$, the following standard equalities hold:

$$
[1 a ; 0 b]=a, \quad[p a ;(1-p) b]=[(1-p) b ; p a], \quad[q[p a ;(1-p) b] ;(1-q) b]=[p q a ;(1-p q) b] .
$$

A preference relation on $M$ is a binary relation $\geq$ with the interpretation that $a \geq b$ means that " $a$ is at least as good as $b "$.

A function $u: M \rightarrow \mathbb{R}$ is an expected utility function representing the preference relation $\geq$ if for all $a, b \in M$ and $p \in[0,1]$, it holds that

$$
\begin{aligned}
& \text { (i) } u(a) \geq u(b) \text { if and only if } a \geq b \text {, and } \\
& \text { (ii) } u([p a ;(1-p) b])=p u(a)+(1-p) u(b) .
\end{aligned}
$$

It is well-known that, if $u$ is an expected utility function representing the preference relation $\geq$, then also every positive affine transformation $\bar{u}$ given by $\bar{u}(a)=\alpha u(a)+\beta$, for some $\alpha>0, \beta \in \mathbf{R}$, 
represents this preference relation. We write $[a>b]$ if $[a \geq b$ and $b \nsucceq a]$, and $[a \sim b]$ if $[a \geq b$ and $b \geq a$ ]. The following axioms guarantee that an expected utility function representing $\geq$ exists.

Axiom 1 (Completeness). For any $a, b \in M$, either $a \geq b$ or $b \geq a$.

Axiom 2 (Transitivity). For any $a, b, c \in M$ such that $a \geq b$ and $b \geq c$, it holds that $a \geq c$.

Axiom 3 (Continuity). For any $a, b, c \in M$, the sets $\{p \mid[p a ;(1-p) b] \geq c\}$ and $\{p \mid c \geq[p a ;(1-p) b]\}$ are closed.

Axiom 4 (Substitutability). If $a, a^{\prime} \in M$ and $a \sim a^{\prime}$, then for every $b \in M$, $\left[\frac{1}{2} a ; \frac{1}{2} b\right] \sim\left[\frac{1}{2} a^{\prime} ; \frac{1}{2} b\right]$.

We assume throughout the paper that preferences satisfy these axioms. We refer to preferences satisfying these axioms as regular preferences.

\section{Centrality and utility in digraphs}

We refer to a pair $(i, \omega) \in N \times \mathcal{W} \mathcal{D}^{N}$ as a digraph position. We assume that a preference relation $\geq$ is defined on the set $N \times \mathcal{W} \mathcal{D}^{N}$ of digraph positions. We interpret $(i, \omega) \geq\left(j, \omega^{\prime}\right)$ as "it is at least as good to be in the position of node $i$ in digraph $\omega$ as to be in the position of node $j$ in digraph $\omega^{\prime \prime}$. Let $M$ be the mixture set generated by all digraph positions $(i, \omega) \in N \times \mathcal{W} \mathcal{D}^{N}$ containing all simple and compound lotteries over digraph positions, lotteries over lotteries, etc. For $(i, \omega),\left(j, \omega^{\prime}\right) \in N \times \mathcal{W} \mathcal{D}^{N}$ and $p \in[0,1]$, the lottery $\left[p(i, \omega) ;(1-p)\left(j, \omega^{\prime}\right)\right]$ considers a type of risk with respect to taking a position in a digraph. It means that with probability $p$ the agent takes the position of node $i$ in digraph $\omega$, and with probability $(1-p)$ he takes the position of node $j$ in digraph $\omega^{\prime}$. We extend the preference relation over digraph positions to the mixture set $M$. Then an expected utility function for digraph positions is a function $\phi: M \rightarrow \mathbf{R}$ assigning a utility value to every mixture of digraph positions satisfying conditions (5).

Inspired by [42], besides the standard axioms on mixture sets stated in the preliminaries (Axioms 1-4), we introduce some axioms that are specific for preferences over digraph positions.

Anonymity (Axiom 5) requires that relabeling the nodes in a digraph yields a corresponding reordering in the preference relation.

Axiom 5 (Anonymity). For all $\omega \in \mathcal{W D}^{N}, i \in N$ and $\pi \in \Pi(N)$, it holds that $(i, \omega) \sim(\pi(i), \pi \omega)$.

The following two axioms, Axioms 6 and 7 below, compare different digraph positions, expressing what is the worst that can happen, and specifying at least one digraph position that is strictly better than a worst position. Regarding what is the worst, we assume that an agent weakly prefers any position in any digraph above being isolated. 
Axiom 6 (Isolated is the worst). For all $i \in N, \omega \in \mathcal{W} \mathcal{D}^{N}$ and $\omega^{\prime} \in \mathcal{W} \mathcal{D}_{i}^{N}$, i.e., $i$ is isolated in $\omega^{\prime}$, it holds that $(i, \omega) \geq\left(i, \omega^{\prime}\right)$.

Notice, that this implies that an agent is indifferent between any two digraphs where he is isolated. Specifically, an agent is indifferent between being in the empty graph $\omega^{0}$ and being isolated in any other digraph. We want to remark that this rules out externalities in the sense that as long as an agent is isolated, he is indifferent with respect to the way the other nodes are connected. Although from an economic viewpoint there might be reason why such externalities exist, this property is satisfied by almost all social network centrality measures.

Next, we assume that it is strictly better to be the center of the outward oriented star graph $\omega^{i}$, than being in the empty graph ${ }^{6}$

Axiom 7 (Center of the star is strictly better than being isolated). For all $i \in N$, it holds that $\left(i, \omega^{i}\right)>$ $\left(i, \omega^{0}\right)$.

Finally, we apply one of the two risk neutrality axioms introduced by [42] for TU-games, namely neutrality to ordinary risk. For $p \in[0,1]$, consider the convex combination $p \omega+(1-p) \omega^{\prime}$ of two digraphs $\omega$ and $\omega^{\prime}$, i.e. $\left(p \omega+(1-p) \omega^{\prime}\right)(i, j)=p \cdot \omega(i, j)+(1-p) \cdot \omega^{\prime}(i, j)$ for all $i, j \in N$. Neutrality to ordinary risk requires that an agent is indifferent between taking a position in digraph $p \omega+(1-p) \omega^{\prime}$ for sure, and playing a lottery over the digraphs $\omega$ and $\omega^{\prime}$ with the corresponding probabilities.

Axiom 8 (Neutrality to ordinary risk). For all $\omega, \omega^{\prime} \in \mathcal{W} \mathcal{D}^{N}$ and $i \in N$, it holds that $(i, p \omega+(1-$ $\left.p) \omega^{\prime}\right) \sim\left[p(i, \omega) ;(1-p)\left(i, \omega^{\prime}\right)\right]$.

Neutrality to ordinary risk reflects a risk neutrality with respect to lotteries in situations where the outcome (in this case the weights of links) is unknown, but the probability distribution over the outcomes is known. As we will see below, this allows an agent to make decisions based on expected utility, which is a common practice in Economics and Operations Research.

It turns out that a utility function for digraph positions that represents regular preferences satisfying the four axioms of this section, must be a linear combination of the outdegree and indegree measures with positive weight on the outdegree and nonnegative weight on the indegree measure. By scale invariance, this implies that such a preference relation can be represented by a convex combination of the outdegree and indegree measures with positive weight on the outdegree.

\footnotetext{
${ }^{6} \mathrm{We}$ could do with the weaker axiom that requires that there is at least one position that is strictly better than being isolated, but in most applications it seems reasonable that this holds for the center of a star.
} 
Theorem 1. The utility function $\phi$ represents a regular preference relation that satisfies anonymity, isolated is the worst, center of the star is strictly better than being isolated, and neutrality to ordinary risk if and only if there exists $\alpha \in(0,1]$ such that $\phi(i, \omega)=\alpha \cdot$ out $_{i}(\omega)+(1-\alpha) \cdot \operatorname{in}_{i}(\omega)$ for all $(i, \omega) \in N \times \mathcal{W} \mathcal{D}^{N}$, where out $i_{i}(\omega)$ and $\operatorname{in}_{i}(\omega)$ are, respectively the outdegree and indegree of node $i$ in digraph $\omega$, see (1) and (2).

The proof of Theorem 1 and all other results of this section, can be found in Appendix B. We show logical independence of the axioms in Theorem 1 in Appendix C.

The class of utility functions/power measures characterized in this theorem contains the outdegree $(\alpha=1)$ and the average of the out- and indegree $\left(\alpha=\frac{1}{2}\right)$ as special cases.$^{7}$

Theorem 1 can be shown by (i) characterizing the linear combinations of the outdegree and indegree measures as those power measures that satisfy four properties of power measures for digraphs (Proposition 1 below), and (ii) relating those four graph measure properties to properties of preference relations over digraph positions (Lemma 1 below).

Note that power measures $f$ and utility functions $\phi$ are mathematically the same and both assign values to nodes in digraphs, but their interpretations are different. Because of this difference in interpretation, we use different notation. In this paper, we bring these interpretations together.

First, anonymity of a power measure means that the labeling of the nodes in a digraph has no effect on their power 8

Property 1 (Anonymity). For every $\omega \in \mathcal{W D}^{N}$ and permutation $\pi \in \Pi(N)$, it holds that $f_{i}(\omega)=$ $f_{\pi(i)}(\pi \omega)$.

Second, the isolated node property requires that the power of an isolated node does not depend on the structure of the rest of the graph. Notice that this implies that for isolated nodes there are no externalities with respect to how other nodes are connected.

Property 2 (Isolated node property). For every $\omega, \omega^{\prime} \in \mathcal{W} \mathcal{D}_{i}^{N}$, i.e., $i \in N$ is isolated in both digraphs, it holds that $f_{i}(\omega)=f_{i}\left(\omega^{\prime}\right)$.

Scale invariance states that if the weights of all arcs in a digraph are multiplied by a common factor, then the powers of the nodes in that digraph are multiplied by the same factor.

\footnotetext{
${ }^{7}$ As shown in the proof of Theorem 1 in Appendix B, $\alpha$ cannot be equal to zero since (i) the utility of a node in the empty digraph equals zero, and (ii) the utility of being the center of the outward oriented star is $\alpha$ times its outdegree (being $|N|-1)$. Therefore, 'center of the star is strictly better than being isolated' (Axiom 7), implies that $\alpha$ should be positive.

${ }^{8}$ For some properties, we use the same name for preference relations as for power measures. The context makes clear if we speak about preferences or power measures.
} 
Property 3 (Scale invariance). Let $\omega \in \mathcal{W D}^{N}$ and $\alpha \in \mathbf{R}_{+}$. Then $f(\alpha \omega)=\alpha f(\omega)$, where $\alpha \omega \in \mathcal{W D}^{N}$ is given by $\alpha \omega(i, j)=\alpha \cdot \omega(i, j)$ for all $i, j \in N, i \neq j$.

Finally, additivity means that the power in the digraph obtained by adding two digraphs, is equal to the sum of the powers of these two digraphs.

Property 4 (Additivity). For $\omega, \omega^{\prime} \in \mathcal{W D}^{N}$, it holds that $f\left(\omega+\omega^{\prime}\right)=f(\omega)+f\left(\omega^{\prime}\right)$, where $(\omega+$ $\left.\omega^{\prime}\right)(i, j)=\omega(i, j)+\omega^{\prime}(i, j)$ for all $i, j \in N, i \neq j$.

These four properties characterize the linear combinations of the outdegree- and indegree measures.

Proposition 1. A power measure $f$ on $\mathcal{W D}^{N}$ satisfies anonymity, the isolated node property, scale invariance and additivity, if and only if $f \in \operatorname{Lin}\{$ out, in\}, i.e., there exist $\alpha, \beta \in \mathbf{R}$ such that

$$
f_{i}(\omega)=\alpha \cdot \operatorname{out}_{i}(\omega)+\beta \cdot \operatorname{in}_{i}(\omega) \text { for all }(i, \omega) \in N \times \mathcal{W} \mathcal{D}^{N} .
$$

Although a further characterization of specific values of $\alpha$ and $\beta$ is beyond the goal of this paper, intuitively, (i) when $\alpha=\beta$ outgoing and ingoing arcs are evaluated equally, which is reasonable when 'connectedness' is the main issue irrespective of the role on the arc, while (ii) with $\beta=0, \alpha>0$ only outgoing arcs are taken into account when evaluating the desirability of graph positions, and thus it fully focusses on 'dominance'.9

Theorem 1 follows from Proposition 1 by the following lemma which shows how the four properties for graph power measures are implied by the axioms on preferences discussed before.

Lemma 1. Consider an expected utility function $\phi: N \times \mathcal{W} \mathcal{D}^{N} \rightarrow \mathbf{R}$ for positions in a digraph that is determined by a power measure $f$ as follows: $\phi(i, \omega)=f_{i}(\omega)$.

(i) If expected utility function $\phi$ represents a regular preference relation $\geq$ satisfying anonymity (Axiom 5), then power measure $f$ satisfies anonymity.

(ii) If expected utility function $\phi$ represents a regular preference relation $\geq$ satisfying isolated is the worst (Axiom 6), then power measure f satisfies the isolated node property.

(iii) If expected utility function $\phi$ represents a regular preference relation $\geq$ satisfying anonymity, isolated is the worst and ordinary risk neutrality (Axioms 5, 6, 8), then $f$ is a power measure that satisfies scale invariance and additivity.

Notice that Proposition 1 and Lemma 1 directly imply that the axioms in Theorem 1 determine that the utility function is a linear combination of the indegree and outdegree measure. The proofs of Proposition 1, Lemma 1 and Theorem 1 can be found in Appendix B.

\footnotetext{
${ }^{9}$ It follows from the proof of Proposition 1 in the appendix that adding score normalization as in [55], requiring that for every digraph the sum of the power values equals the sum of the weights over all arcs in the digraph, implies that $\beta=1-\alpha$.
} 
Our main result (Theorem 1) gives an interpretation of power measures for digraphs as von Neumann-Morgenstern expected utility functions. Using Lemma 1, this follows from Proposition 1 which is a social network result that characterizes the (linear combinations of the) outdegree- and indegree measures as a digraph power measure. Lemma 1 'bridges' social network theory with economic utility theory in the sense that properties of preference relations are related to properties of power measures representing these preferences.

In Section 5, we implement the axioms on preference relations and power measures in terms of broadcasting games, queueing games and telecommunication games. First, in the next section we consider undirected graphs.

\section{Centrality and utility in undirected graphs}

Next, we consider the subclass of undirected graphs, and interpret centrality measures for undirected graphs as von Neumann-Morgenstern expected utility functions. We refer to a pair $(i, \omega) \in N \times \mathcal{W} \mathcal{G}^{N}$ as a (undirected) graph position. Similar as in the previous section on digraphs, we assume that a preference relation $\geq$ is defined on the set $N \times \mathcal{W} \mathcal{G}^{N}$ of undirected graph positions, and interpret $(i, \omega) \geq\left(j, \omega^{\prime}\right)$ as "it is at least as good to be in the position of node $i$ in graph $\omega$ as to be in the position of node $j$ in graph $\omega^{\prime \prime}$. Let $M$ again be the mixture set generated by all undirected graph positions $(i, \omega) \in N \times \mathcal{W} \mathcal{G}^{N}$ containing all simple and compound lotteries over graph positions. Preference relations $\geq$, expected utility functions $\phi: M \rightarrow \mathbf{R}$ and lotteries $\left[p(i, \omega) ;(1-p)\left(j, \omega^{\prime}\right)\right]$, are defined similar as in the previous section for the more general directed graphs.

Besides the standard axioms stated in the preliminaries, we use the axioms of the previous section, but applied to undirected graphs. For completeness, we explicitly give the axioms for undirected graphs, although three of them are obtained by directly applying the corresponding axioms of the previous section by putting for each link $\{i, j\}$ two arcs, $(i, j)$ and $(j, i)$, oriented in opposite directions.

Axiom 9 (Anonymity). For all $\omega \in \mathcal{W G}^{N}, i \in N$ and $\pi \in \Pi(N)$, it holds that $(i, \omega) \sim(\pi(i), \pi \omega)$.

Axiom 10 (Isolated is the worst). For all $i \in N, \omega \in \mathcal{W} \mathcal{G}^{N}$ and $\omega^{\prime} \in \mathcal{W G}_{i}^{N}$, i.e., $i$ is isolated in $\omega^{\prime}$, it holds that $(i, \omega) \geq\left(i, \omega^{\prime}\right)$.

Axiom 11 (Center of the star is strictly better than being isolated). For all $i \in N$, it holds that $\left(i, \omega^{i}\right)>$ $\left(i, \omega^{0}\right)$.

Axiom 12 (Neutrality to ordinary risk). For all $\omega, \omega^{\prime} \in \mathcal{W} \mathcal{G}^{N}$ and $i \in N$, it holds that (i, $p \omega+(1-$ p) $\left.\omega^{\prime}\right) \sim\left[p(i, \omega) ;(1-p)\left(i, \omega^{\prime}\right)\right]$.

Notice that Axioms 9, 10 and 12 are stated exactly the same as the corresponding axioms in the previous section, and are direct applications of these axioms to the special class of undirected graphs. 
Regarding Axiom 11, the outward oriented star in directed graphs, that is used in Axiom 7, cannot be represented as an undirected graph, and alternatively is replaced by the star in undirected graphs. ${ }^{10}$

The main result of this section characterizes the degree measure for undirected graphs as expected utility function that represents a regular preference relation that is neutral to ordinary risk.

Theorem 2. A preference relation $\geq$ over graph positions $N \times \mathcal{W G} \mathcal{G}^{N}$ is regular and satisfies anonymity, isolated is the worst, center of the star is strictly better than being isolated, and neutrality to ordinary risk if and only if it can be represented by utility function $\phi(i, \omega)=d_{i}(\omega)$ for all $(i, \omega) \in N \times \mathcal{W G}$, where $d_{i}(\omega)$ is the degree of node $i$ in graph $\omega$, see (3).

This theorem gives the degree measure, which is a well-known centrality measure in social network theory, an interpretation as a von Neumann-Morgenstern expected utility function. Similarly as in the previous section, Theorem 2 can be shown by (i) characterizing the multiples of the degree measure as those centrality measures that satisfy four properties of centrality measures for graphs (Proposition 2 below), and (ii) relating those four graph centrality properties to properties of preference relations over graph positions (Lemma2 2 below). Although the results of this section do not follow as a corollary of [42], by realizing that graphs form a special class of cooperative TU-games, the proofs are very close to those of [42]. Therefore, we omit the proofs of this section ${ }^{11}$

A centrality measure $f$ on $\mathcal{W G}^{N}$ satisfies:

- anonymity if for every $\omega \in \mathcal{W G}^{N}$ and permutation $\pi \in \Pi(N)$, it holds that $f_{i}(\omega)=f_{\pi(i)}(\pi \omega)$;

- scale invariance if for every $\omega \in \mathcal{W} \mathcal{G}^{N}$ and $\alpha \in \mathbf{R}_{+}$, it holds that $f(\alpha \omega)=\alpha f(\omega)$ where $\alpha \omega \in$ $\mathcal{W G}^{N}$ is given by $\alpha \omega(\{i, j\})=\alpha \cdot \omega(\{i, j\})$ for all $\{i, j\} \in L^{c}$;

- isolated node property if for every $\omega, \omega^{\prime} \in \mathcal{W} \mathcal{G}_{i}^{N}$, it holds that $f_{i}(\omega)=f_{i}\left(\omega^{\prime}\right)$;

- additivity if for every $\omega, \omega^{\prime} \in \mathcal{W} \mathcal{G}^{N}$, it holds that $f\left(\omega+\omega^{\prime}\right)=f(\omega)+f\left(\omega^{\prime}\right)$, where $\left(\omega+\omega^{\prime}\right)(\{i, j\})=$ $\omega(\{i, j\})+\omega^{\prime}(\{i, j\})$ for all $\{i, j\} \in L^{c}$.

Proposition 2. A centrality measure $f$ satisfies anonymity, the isolated node property, scale invariance and additivity if and only if there exists an $\alpha \in \mathbf{R}$ such that

$$
f_{i}(\omega)=\alpha d_{i}(\omega) \text { for all }(i, \omega) \in N \times \mathcal{W G}^{N} .
$$

The four properties in Proposition 2 are similar to the anonymity, null player, scale invariance and additivity properties that, together with efficiency, characterize the Shapley value for TU-games. Since

\footnotetext{
${ }^{10}$ Again, we could do with a weaker version of Axiom 11 requiring that there is at least one graph position that is strictly better than being isolated. This is often required when measuring centrality in the social network literature, see e.g. [25].

${ }^{11}$ The proofs of this section can be obtained from the authors on request.
} 
the Shapley value of the 2-additive game associated to a graph (see Appendix A) assigns to every player half of its degree (see [19], Theorem 1), this proposition can be interpreted as saying that Shapley's axiomatization is also valid on the subclass of 'graph games'. This is, for example, not true for other subclasses of games such as, e.g., simple games, assignment games ([58]), etc.

Theorem 2 follows from Proposition 2 by the following lemma which shows how the four properties for graph centrality measures are implied by the Axioms 9-12 on preferences.

Lemma 2. Consider an expected utility function $\phi: M \rightarrow \mathbf{R}$ for positions in a graph that is determined by a centrality measure $f$ as follows: $\phi(i, \omega)=f_{i}(\omega)$.

(i) If expected utility function $\phi$ represents a preference relation $\geq$ satisfying anonymity (Axiom 9), then centrality measure $f$ satisfies anonymity.

(ii) If expected utility function $\phi$ represents a preference relation $\geq$ satisfying isolated is the worst (Axiom $[10$, then centrality measure $f$ satisfies the isolated node property.

(iii) If expected utility function $\phi$ represents a preference relation $\geq$ satisfying anonymity, isolated is the worst and ordinary risk neutrality (Axioms 90,12 ), then $f$ is a centrality measure that satisfies scale invariance and additivity.

Notice that Proposition 2 and Lemma 2 directly imply that the axioms in Theorem 2 determine that the utility function is a multiple of the degree measure.

Similarly as for directed graphs, Theorem 2 interprets centrality measures as von NeumannMorgenstern expected utility functions. Using Lemma 2, this follows from Proposition 2 which is a social network result that characterizes the (multiples of the) degree measure as a graph centrality measure. In this sense, Lemma2 'bridges' social network theory with economic utility theory.

\section{Applications}

Cooperative games where worth is generated by cooperation in two-player coalitions, referred to as 2-additive games, have many applications in Economics and Operations Research. Examples are broadcasting games (see [6]), queueing games (see [33]) and telecommunication games (see [60]). In this section, we discuss the implication of our results on these three applications.

In all these applications, the Shapley value plays an important role. For 2-additive games, the Shapley value coincides with several other game theory solutions such as the nucleolus ([48]), the $\tau$ value ([52]) and the proportional allocation of nonseparable cost ([34]), see [60]. As argued by [19], for undirected graphs, the Shapley value coincides with the degree measure.

To have a uniform notation in this paper, we put all notions in this section in terms of weighted digraphs which is an equivalent representation as the models in the mentioned literature. We prefer the 
notation of weighted graphs and digraphs, since these are very well-known models in the literature, in particular, in the Operations Research literature ${ }^{12}$

\subsection{Broadcasting games}

The broadcasting games of [6] consider soccer competitions where every team plays twice against every other team, once at each team's home stadium. The league of all teams has to decide how to share the revenues from broadcasting rights among the teams. The teams are represented by the set $N$, and the revenues of the match between teams $i$ and $j$ at the home stadium of team $i$ are given by weight $\omega(i, j)$. One of the allocation rules considered by [6] is the equal-split rule that is given by $E S_{i}(N, \omega)=$ $\sum_{j \in N \backslash\{i\}} \frac{\omega(i, j)+\omega(j, i)}{2}$ for all $i \in N{ }^{13}$

They show that this rule is the unique rule that satisfies:

(i) Equal treatment of equals: $f_{i}(\omega)=f_{j}(\omega)$ if $\omega(i, k)=\omega(j, k)$ and $\omega(k, i)=\omega(k, j)$ for all $k \in N \backslash\{i, j\}$;

(ii) Additivity: is defined the same as in Sections 3 and 4 , see Property 4

(iii) Null team: $f_{i}(\omega)=0$ if $i$ is isolated (i.e. if $\omega(i, k)=\omega(k, i)=0$ for all $k \in N \backslash\{i\}$ ).

Comparing these axioms with the axioms in Proposition 1, we can conclude that:

(i) Equal treatment of equals is not related to anonymity. Equal treatment of equals as defined by [6] is rather strong since it requires equal payoffs between two players, even when there is a difference between the revenues of the home and away match between them. This generates that, if we only consider the two matches $(i, j)$ and $(j, i)$, the two teams $i$ and $j$ are 'equal', and thus equally share the sum of the revenues of the two matches between the two of them (since by null team the other teams get zero share in this). As follows from Proposition 1, our axioms allow an unequal sharing between the home and away team.

(ii) Additivity is a standard axiom for allocation rules and graph power measures.

\footnotetext{
${ }^{12}$ In this section we state several claims without proof, since they all follow as corollaries from, or small variations of, the results in this paper. Explicit proofs are available from the authors on request.

${ }^{13}$ Although this situation is represented by a digraph, [6] shows that this value coincides with the Shapley value (and thus nucleolus, $\tau$-value, proportional division of nonseparable cost) of the associated 2-additive standard game where the worth of every coalition equals the sum of the weights between teams in the coalition. Alternatively, this value can be obtained as the value of [47] of the associated generalized characteristic function game (see [38]) where the worth of every ordered coalition equals the sum of the weights of the arcs oriented in the direction of this ordered coalition, between teams in this coalition.
} 
(iii) Null team requires zero payoffs for teams that generate zero revenues. Our isolated node property is weaker and only requires that the teams $k \in N \backslash\{i, j\}$ all get the same share in the revenues of the matches between $i$ and $j$, and this share is at most equal to the share of $i$ and $j$. However, together with additivity, this share is zero (see the proof of Proposition 1 in the appendix).

Our axioms allow any sharing of the revenue of the matches between $i$ and $j$ among these two players. However, the shares $\alpha$ and $\beta$ that are assigned to the home and away team are the same over all matches in the competition (again, see the proof of Proposition 11.

If we add the normalization used by [6], namely that $\sum_{i \in N} f_{i}(\omega)=\sum_{i, j \in N} \omega(i, j)$, then we conclude that $\beta=1-\alpha$, and thus the revenues of every match are shared exactly between the two teams who play the match. Concluding, Proposition 1 applied to broadcasting games, together with the normalization, characterizes the class of rules that share the revenue of every match between the two teams who play the match, where for each match the home team gets share $\alpha \in[0,1]$ and the away team gets share $1-\alpha$ in the revenues of this match. Obviously, the equal-split rule is obtained by taking $\alpha=\frac{1}{2}$.

A preference relation $\geq$ on the teams in a broadcasting game, can be interpreted as saying $(i, \omega) \geq\left(j, \omega^{\prime}\right)$ if and only if team $i$ in competition $\omega$ is 'more profitable' than team $j$ in competition $\omega^{\prime}$. Considering the interpretation of our class of rules, and specifically the equal-split rule, as utility function representing such preferences, we cannot only compare teams within one competition, but also teams in different competitions. For example, we can compare Real Madrid (say team $i$ ) in the Spanish Football League (say competition $\omega$ ) with Liverpool Football Club (say team $j$ ) in the English Premier League (say competition $\omega^{\prime}$ ). Comparing the profitability of teams in sport competitions can be useful to determine the attractiveness of these teams from the viewpoint of the revenues. For example, this can help investors who want to invest in such teams, or when a league wants to determine what is a 'fair' way to allocate the revenues, where this fairness is determined by the underlying axioms.

Translating our axioms 1-4 in terms of broadcasting games, we obtain:

(i) Anonymity has the usual interpretation, implying indifference between two teams that take similar positions.

(ii) Isolated is the worst requires that a team such that none of its matches generates any revenue, should be the least profitable (which is implied when using [6]'s Null team axiom).

(iii) Center of the star is strictly better than being isolated implies that, if there is a team such that every match with positive revenue involves this team (being an essential team in the terminology of [6]), then this team should be more profitable than a null team that generates no revenue. This 
is implied by [6]'s stronger essential team and null team axiom which requires that such a team gets all revenues ${ }^{14}$

(iv) Neutrality to ordinary risk considers situations of risk with respect to the revenues. Suppose that beforehand it is not known what will be the revenues, but the probability distribution over the possible outcomes is known. For example, the revenues might depend on weather conditions or maybe the contract with the broadcasting company will be renewed or it might happen that for some reason not all scheduled matches in the competition will be played. Neutrality to ordinary risk implies that, in that case, one can compare the profitability of different teams in one competition by comparing them in the 'expected' competition where the revenues of all matches are simply obtained as the expected value of these revenues under the given probability distribution.

\subsection{Queueing games}

The queueing games of [33] describe situations where a set of agents are waiting to be served on a machine. Each agent $i \in N$ has a fixed nonnegative unit waiting $\operatorname{cost} \theta_{i}$, and the machine can only serve one agent at a time. It is efficient to give priority to agents with a higher waiting cost (assuming all agents have the same processing time), and the question is how agents that get priority (i.e. the agents with high waiting cost) should compensate agents that have to wait longer in the queue (i.e. agents with lower waiting cost). An allocation assigns to each agent a position in the queue and a (positive or negative) monetary transfer. A rule assigns an allocation to every queueing problem. Assuming quasilinear utility functions, where the utility of an agent for an allocation is the sum of its total waiting cost (depending on its position in the queue) and the monetary transfer, the literature considers 'fair' rules for queueing problems. One of the most famous rules is the minimal transfer rule of [33], which associated utility function is obtained as the Shapley value of the queueing game where the worth of a coalition equals the minimal total cost for the agents in this coalition (i.e. the cost if they are in an efficient queue among themselves) if they are served before all other agents. Since these games are 2 -additive games, where the worth of a two-player coalition equals the minimum of the waiting costs of the two agents in this coalition (see Lemma 1 in [33]), the minimal transfer rule is also obtained as the nucleolus, $\tau$-value, or proportional division of nonseparable cost of the associated queueing game.

The preference relation expresses preferences over being in different queueing problems with different waiting costs. Beforehand, it is not clear what would be a better queue to be in. On one hand,

\footnotetext{
${ }^{14}$ Obviously, the equal-split rule does not satisfy the stronger essential team version in [6], who use this to characterize the alternative concede-and-divide rule.
} 
having a high (respectively low) waiting cost has the advantage (respectively disadvantage) that one will be served earlier (respectively later) in the queue. But on the other hand, having a high (respectively low) waiting cost has the disadvantage (respectively advantage) that one has to pay a higher monetary transfer (respectively will receive a higher monetary transfer). For this application, the axioms of Theorem 2 can be interpreted as follows (where we take into account that this is a cost allocation game):

(i) Anonymity has the usual interpretation, implying indifference between agents with the same waiting costs in queues with the same waiting cost distribution.

(ii) An agent is isolated if and only if (i) its cost is zero, or (ii) it is the only agent with a positive waiting cost (in which case all agents are isolated). Isolated is the worst requires that such an agent should have the lowest contribution in the cost. This expresses that an agent with zero waiting cost can be asked to contribute to the total cost of the queue, but cannot be exploited in the sense that it should always be an agent with the lowest contribution. In case an agent is the only agent with a positive waiting cost, since he should also not contribute more than the other agents, this implies that the positive cost agent is served first, and there are no compensations for the other agents, who can wait for zero cost.

(iii) The only star graph that is possible in this case, is a star with only one link, which means that there are exactly two agents with positive waiting cost ${ }^{15}$ Center of the star is strictly better than being isolated, then implies that the two agents with positive waiting cost contribute at least as much (in terms of transfer and position in the queue) as the agents with zero waiting cost. (Notice that the total cost in a queue with at least two agents with positive waiting cost is positive.)

(iv) Neutrality to ordinary risk again considers situations of risk, now with respect to the waiting costs of all agents. Similar to broadcasting games, suppose that beforehand it is not known what will be the waiting costs in the queue, but the probability distribution over the possible outcomes is known. Then, one can compare the desirability of different agents in a queue by comparing them in the 'expected' queue where the waiting costs are simply obtained as the expected value of the waiting costs under this probability distribution.

Our main result implies that the minimal transfer rule is the only utility function that represents such preferences, and thus can be seen as a vNM expected utility function if the axioms underlying the

\footnotetext{
${ }^{15}$ If there are two links with positive weight, say $\{i, j\}$ and $\{i, h\}$, then the cost of all three agents must be positive, and therefore also the weight of link $\{j, h\}$ is positive.
} 
preferences are satisfied. Since the class of queueing games form a proper subclass of 2-additive games, it is not obvious that our axioms characterize the class of rules as described in Proposition 2 .

\subsection{Telecommunication games}

The telecommunication games of [60] describe situations where operators of the Terrestrial Flight Telephone System (TFTS) use each other's ground stations and decide how to share the revenues from in flight telephone calls. In the associated coalitional game, the players are the countries whose national operators participate in the cooperation. Each country invests in ground stations to cover its area, and in apparatus in airplanes of airlines that have their home base in this country. The contribution of a specific country to the amount of telephone calls that are made using TFTS consists of two parts: (i) the telephone calls that are provided with the service by the country itself, and (ii) the telephone calls that are made from airplanes flying over the country. We can model this by $\omega(i, j)$ being the revenues of telephone calls made by airplanes of country $j$ that are using the ground stations at country $i$. Notice that in this application, the graph is not irreflexive, i.e. $\omega(i, i)$ can be positive if revenues are made by country $i$ 's own airplanes flying over $i$ 's own territory. As a result the associated TFTS game is the sum of an inessential (or 1-additive) game $e^{16}$ and a 2-additive game. However, we can straightforward apply our results also in this case 17

In this application, a preference relation reflects the position of countries in this revenue sharing problem. For ease of interpretation, we assume that all internal revenues are zero, i.e $\omega(i, i)=0$ for all $i \in N$. The axioms of Theorem 2 can be interpreted as follows:

(i) Anonymity again has the usual interpretation, implying indifference between similar positions regarding the use of other countries' ground stations.

(ii) A country is an isolated node if and only if its airplanes do not use other countries ground station, and its own ground station is not used by foreign airplanes. Isolated is the worst then requires that such a country should have the lowest share in the revenues (besides its own internal revenues).

(iii) A country is a center of the star if and only if this country is the unique country that generates revenue with other countries (either by using the ground stations of other countries, or other countries' using its ground station). Center of the star is strictly better than being isolated says that

\footnotetext{
${ }^{16} \mathrm{An}$ inessential or 1 -additive game is a game where the worth is determined by singletons, i.e. $v(S)=\sum_{i \in S} v(\{i\})$ for all $S \subseteq N$.

${ }^{17}$ This is because of additivity of the Shapley value, and thus it assigns to a TFTS game simply the worth of a country in the 1 -additive game and its Shapley value in the 2 -additive game.
} 
such a country is strictly better off than a country that does not generate any revenue with other countries (again ignoring internal revenues).

(iv) Neutrality to ordinary risk considers situations of risk with respect to the phone calls made in flights. Suppose that beforehand it is not known what phone calls will be made, but the probability distribution over the possible outcomes is known. Then one can compare the profitability of different countries by comparing them in the 'expected' situation where the revenues are based on the expected number of phone calls that are made.

\section{Concluding remarks}

The goal of this paper is to (i) give a utility foundation to centrality measures, and in that way to connect social network theory with economic utility theory, (ii) show that [42]'s utility foundation of the Shapley value also holds on the smaller class of undirected graphs or 2-additive games with applications such as telecommunication games and queueing games, and (iii) to extend these results to digraphs which include applications such as broadcasting (revenue sharing) games. This paper can be seen as a starting point to build such a utility foundation of graph centrality. Since economic decision making is based on preferences of economic decision makers, a utility foundation is fundamental for the application of graph centrality measures in economic models.

A next step would be to analyze (economic) processes on a network and to combine utility of positions in a network with utility generation from processes on a network. Also, we remark that, although we assumed the set of nodes to be fixed, we can still analyze networks of different size, since we did not assume the network to be connected. So, we can compare 'large' networks (for example connecting all the nodes) with 'small' networks (when we have many components).

The approach followed in this paper is of crucial importance, since the interpretation of the degree measure as expected utility function for graph positions permits to compare different positions in different graphs. Despite its simplicity, the degree measure is sufficient for measuring involvement or communication ability of an agent in the graph. Moreover, the simplicity of the degree measure is an advantage, since only the local structure around a node must be known for calculations, for instance, when using social survey data. Our utility interpretation is also an alternative to the usual interpretation of the degree as either a myopic centrality measure that only takes account of direct relations, or as a farsighted centrality measure in the sense that for a simple connected graph, the probability that a random walk will be found at node $i$ in the limit of long time is proportional to the degree of that node, see e.g., [36]. Moreover, in applications of 2-additive games, the degree measure is equivalent to the Shapley value 
which, on that class of games coincides with several other well-known solutions such as the nucleolus, $\tau$-value and proportional allocation of nonseparable costs.

Besides its applications to Economics and Operations Research, the present paper is also related to the literature on social networks and centrality; for surveys see, e.g. [23], [30], and [36].

Axiomatic approaches to the degree measure can be found in, e.g. [24], [12], and [55], whereas [7] characterizes the standard centrality measures within a unified framework and shows that they all are characterized by a common set of axioms.

Besides considering the degree measure (or Shapley value) as a centrality measure for graphs (or allocation rule for 2-additive games), we mainly considered them as utility function representing preferences over graph (or player) positions. This generalizes the idea of ranking of nodes in a graph. Formally, a ranking method assigns to every (weighted) graph a (complete) preorder on the set of nodes.

Ranking methods based on (out- or in-) degree are characterized in, e.g., [45] (for the ranking by outdegree on the class of tournaments), [13], [56] (for the ranking by outdegree for arbitrary digraphs), and [57] (for the outflow ranking method for weighted digraphs). [21] investigates ranking of items in a graph determined by choice of utility function, whereas the network nodes are used for the ranking. [17, 18] characterizes ranking methods based on evaluations or citations. An important difference between such ranking methods and the topic of the underlying paper is that ranking methods only compare the positions in one and the same network. This is useful if one wants to rank, for example, teams in a sports competition, alternatives in a preference relation, web pages on the internet, etc. Besides such comparisons within one network, a main goal of the underlying paper is to compare positions in different networks. For example, we want to know if an agent prefers a 'central' position in a small network to a position in the fringe of a large network. In order to answer these questions we need to be able to compare positions in different networks. As mentioned before, although we assumed the set of nodes to be fixed, we can compare positions in different networks, since we did not assume the network to be connected, and thus one can compare positions in network components of different size.

We plan a number of follow-up research projects. We intend to relax the assumption of risk neutrality to find utility foundations of other centrality measures. Another issue is how to incorporate externalities in measuring network centrality, allowing that an agent's utility depends on the way how other agents are connected. As mentioned in Sections 3 and 4 , it is very common for social network centrality measures to ignore these externalities. But also in economics, as benchmark cases, often externality-free solutions are proposed such as the externality-free solution for partition function form games in [16], and the externality-free solutions for river water sharing in [1]. 
Considering digraphs, we can consider more possibilities about what is the worst possible position, even when there are no externalities. For example, under the regularity axioms of this paper, the utility function assigns zero to every isolated node in any network. For undirected graphs, it seems reasonable to assume that being isolated is the worst position (as reflected by Axiom 10 ). However, for digraphs, it is not obvious whether it is worse to be isolated or to be connected, but having only ingoing arcs. For broadcasting games the assumption of the underlying paper seems reasonable. But, for example, in social choice theory the famous Copeland score ([15]) (being the difference between the outdegree and indegree) assumes a negative effect of a higher indegree. Also for broadcasting games, by relaxing some assumptions we can provide a utility foundation for other rules than the equal-split rule, such as the concede-and-divide rule that is also considered by [6]. Besides these theoretical research extensions, we plan to do an experimental study, both testing measures of centrality with and without externalities.

\section{Appendix A: The Shapley value and cooperative 2-additive games}

A situation in which a finite set of players $N \subset \mathbb{N}$ can generate certain payoffs by cooperation can be described by a cooperative game with transferable utility (or simply a TU-game), being a pair $(N, v)$ where $v: 2^{N} \rightarrow \mathbb{R}$ is a characteristic function on $N$ satisfying $v(\emptyset)=0$. For every coalition $S \subseteq N$, $v(S) \in \mathbb{R}$ is the worth of coalition $S$, i.e. the members of coalition $S$ can obtain a total payoff of $v(S)$ by agreeing to cooperate. Since the set of players/nodes is fixed, we represent a TU-game $(N, v)$ by its characteristic function $v$.

A payoff vector for game $v$ on $N$ is an $|N|$-dimensional vector $x \in \mathbb{R}^{N}$ assigning a payoff $x_{i} \in \mathbb{R}$ to any player $i \in N$. A (single-valued) solution for TU-games is a function $f$ that assigns a payoff vector $f(v) \in \mathbb{R}^{N}$ to every TU-game $v$ on $N$. One of the most famous solutions for TU-games is the Shapley value ([49]) given by

$$
S_{i}(v)=\sum_{S \subseteq N: i \in S} \frac{(|S|-1) !(|N|-|S|) !}{|N| !}(v(S)-v(S \backslash\{i\})) .
$$

[19] argues that any undirected graph can be represented by a 2-additive TU-game $v$, where the worth of any two player coalition is the weight of the link, and the worth of any other coalition equals the sum of the worths of all two player subcoalitions. In other words, the TU-game $v^{\omega}$ associated to undirected graph $\omega \in \mathcal{W G}^{N}$ is given by $v^{\omega}(S)=\sum_{T \subseteq S,|T|=2} v(T){ }^{18}$ As [19] shows, the Shapley value of

\footnotetext{
${ }^{18}$ In terms of the so-called Harsanyi dividends ([? ]) this can also be defined as the class of games where only two-player coalitions have a nonzero dividend being equal to the weight of the corresponding link.
} 
the associated game $v^{\omega}$ assigns to every player half of its degree in $\omega$. Moreover, [60] and [14] show that on this class of games, the Shapley value coincides with other TU-game solutions such as the nucleolus ([48]) and the $\tau$-value ([52]).

Whereas undirected graphs can be seen as a special subclass of TU-games, this is not the case for digraphs. Without going into details, digraphs can be considered as a special class of cooperative games in generalized characteristic function form where the worth of a coalition depends on the order in which the players enter the coalition. From the various extensions of the Shapley value to generalized TU-games, the outdegree measure is obtained by applying the extension of the Shapley value by [38], while the average of the outdegree and indegree is obtained by applying the alternative extension of the Shapley value by [47].

\section{Appendix B: Proofs of Section 3}

In this appendix, we give the proofs of Section 3. Although the proofs follow similar steps as [42], this cannot be seen as an application of this paper since digraphs cannot be represented by cooperative TU-games.

\section{Proof of Proposition 1}

It is straightforward to verify that power measures as given by (6) satisfy the four properties. To show uniqueness, suppose that power measure $f$ satisfies the four properties, and consider $\omega \in \mathcal{W} \mathcal{D}^{N}$.

First, consider the empty graph $\omega^{0}$ and any graph $\omega \in \mathcal{W D}^{N}$. Additivity implies that $f_{i}\left(\omega+\omega^{0}\right)=$ $f_{i}(\omega)+f_{i}\left(\omega^{0}\right)$. Since $\omega+\omega^{0}=\omega$, this implies that $f_{i}(\omega)=f_{i}(\omega)+f_{i}\left(\omega^{0}\right)$, and thus $f_{i}\left(\omega^{0}\right)=0$ for all $i \in N$. By the isolated node property, $f_{i}(\omega)=0$ for all $\omega \in \mathcal{W D}_{i}^{N}$ where $i$ is isolated.

Next, take a pair $i, j \in N, i \neq j$, and define $\mathcal{W} \mathcal{D}_{i j}^{N}=\left\{\omega \in \mathcal{W} \mathcal{D}^{N} \mid \omega(i, j) \neq 0\right.$ and $\omega(h, g)=0$ for all $(h, g) \neq(i, j)\}$, being the class of graphs where only arc $(i, j)$ has a nonzero weight. By the isolated node property, $f_{k}(\omega)=0$ for all $k \in N \backslash\{i, j\}$. By scale invariance, there exist $\alpha, \beta \in \mathbf{R}$ such that $f_{i}(\omega)=\alpha \cdot \omega(i, j)$ and $f_{j}(\omega)=\beta \cdot \omega(i, j)$ for any $\omega \in \mathcal{W} \mathcal{D}_{i j}^{N}$.

Now take any $(h, g) \in N \times N, h \neq g,(h, g) \neq(i, j)$, and $\omega^{\prime} \in \mathcal{W} \mathcal{D}_{h g}^{N}$. By anonymity and the class $\mathcal{W} \mathcal{D}_{i j}^{N}$ discussed above, we have $f_{h}\left(\omega^{\prime}\right)=\alpha \cdot \omega^{\prime}(h, g), f_{g}\left(\omega^{\prime}\right)=\beta \cdot \omega^{\prime}(h, g)$ and $f_{k}\left(\omega^{\prime}\right)=0$ for all $k \in N \backslash\{h, g\}$. Finally, consider any $\omega \in \mathcal{W} \mathcal{G}^{N}$. For every $i, j \in N, i \neq j$, define $\omega^{i j}(i, j)=\omega(i, j)$ and $\omega^{i j}(h, g)=0$ for all $(h, g) \neq(i, j)$. Then, additivity implies that for all $i \in N, f_{i}(\omega)=\sum_{\substack{h, g \in N \\ h \neq g}} f_{i}\left(\omega^{h g}\right)=\sum_{j \in N \backslash\{i\}}\left(f_{i}\left(\omega^{i j}\right)+f_{i}\left(\omega^{j i}\right)\right)=$ $\sum_{j \in N \backslash\{i\}}(\alpha \cdot \omega(i, j)+\beta \cdot \omega(j, i))=\alpha \sum_{j \in N \backslash\{i\}} \omega(i, j)+\beta \sum_{j \in N \backslash\{i\}} \omega(j, i)=\alpha \cdot \operatorname{out}_{i}(\omega)+\beta \cdot \operatorname{in}_{i}(\omega)$. 


\section{Proof of Lemma 1}

(i) This follows immediately from Axiom 5 .

(ii) If expected utility function $\phi$ represents a preference relation $\geq$ satisfying Axiom 6 , then for every $\omega, \omega^{\prime} \in \mathcal{W D}_{i}^{N}$, we have $(i, \omega) \sim\left(i, \omega^{\prime}\right)$, and thus $f_{i}(\omega)=\phi(i, \omega)=\phi\left(i, \omega^{\prime}\right)=f_{i}\left(\omega^{\prime}\right)$.

(iii) Consider $\omega \in \mathcal{W} \mathcal{D}^{N}$ and $c>1$. Suppose that $\geq$ satisfies neutrality to ordinary risk. Taking $p=\frac{1}{c}$, $\omega^{\prime}=\omega^{0}$ and considering the graph $c \omega$, neutrality to ordinary risk implies that

$$
\left(i,\left(\frac{1}{c} c \omega+\left(1-\frac{1}{c}\right) \omega^{0}\right)\right) \sim\left[\frac{1}{c}(i, c \omega) ;\left(1-\frac{1}{c}\right)\left(i, \omega^{0}\right)\right]
$$

which is equivalent to

$$
(i, \omega) \sim\left[\frac{1}{c}(i, c \omega) ;\left(1-\frac{1}{c}\right)\left(i, \omega^{0}\right)\right] .
$$

Now, let $u$ be a utility function representing a preference relation $\geq$ satisfying Axioms 14 from the preliminaries. From [29], it follows that there exist $r_{0}, r_{1} \in M$ with $r_{1}>r_{0}$ such that an expected utility function $\phi$ over the positions in a digraph $\omega$ can be written as

$$
\phi(i, \omega)=\frac{p_{a b}(i, \omega)-p_{a b}\left(r_{0}\right)}{p_{a b}\left(r_{1}\right)-p_{a b}\left(r_{0}\right)}
$$

for some $a, b \in M$ with $a \geq(i, \omega) \geq b$ and $a \geq r_{1}>r_{0} \geq b$ with probabilities $p_{a b}(i, \omega)$ defined such that $(i, \omega) \sim\left[p_{a b}(i, \omega) a ;\left(1-p_{a b}(i, \omega)\right) b\right]$.

By Axiom 6, we can take $b=r_{0}=\left(i, \omega^{0}\right)$, and thus $p_{a b}\left(r_{0}\right)=p_{a b}\left(i, \omega^{0}\right)=0$ for all $a \in M$.

To show that the preference relation can be represented by a scale invariant power measure, we distinguish the following two cases with respect to $(i, \omega) \in \mathcal{W D}^{N}$ and $c>1$.

Case 1: Suppose that $(i, c \omega) \geq r_{1} 19$

Take $a=(i, c \omega)$. Then by $99, \phi(i, c \omega)=\frac{p_{a b}(i, c \omega)}{p_{a b}\left(r_{1}\right)}=\frac{p_{a b}(a)}{p_{a b}\left(r_{1}\right)}=\frac{1}{p_{a b}\left(r_{1}\right)}$.

By 8 , we have $(i, \omega) \sim\left[\frac{1}{c}(i, c \omega) ;\left(1-\frac{1}{c}\right)\left(i, \omega^{0}\right)\right]$, so $p_{a b}(i, \omega)=\frac{1}{c}$. But then $f_{i}(\omega)=\phi(i, \omega)=\frac{p_{a b}(i, \omega)}{p_{a b}\left(r_{1}\right)}=$ $\frac{1}{c} \cdot \frac{1}{p_{a b}\left(r_{1}\right)}=\frac{1}{c} \phi(i, c \omega)=\frac{1}{c} f_{i}(c \omega)$. So, scale invariance is satisfied in this case.

Case 2: Suppose that $r_{1} \geq(i, c \omega)$.

Take $a=r_{1}$. Then $p_{a b}\left(r_{1}\right)=1$, and so $\phi(i, c \omega)=p_{a b}(i, c \omega)$. By 8 , we have $(i, \omega) \sim\left[\frac{1}{c}(i, c \omega) ;(1-\right.$ $\left.\left.\frac{1}{c}\right)\left(i, \omega^{0}\right)\right] \sim\left[\frac{1}{c}\left[p_{a b}(i, c \omega) a ;\left(1-p_{a b}(i, c \omega)\right) b\right] ;\left(1-\frac{1}{c}\right)\left(i, \omega^{0}\right)\right]=\left[\frac{1}{c} p_{a b}(i, c \omega) a ;\left(1-\frac{1}{c} p_{a b}(i, c \omega)\right) b\right]$, where

\footnotetext{
${ }^{19}$ Although [42] takes specifically that $r_{1}$ is the unanimity game with player $i$ as only nonnull player, we do not specify $r_{1}$. It is sufficient that there exists a graph position that is strictly preferred to being in the empty graph, as is guaranteed by Axiom 7 with the center of the outward oriented star graph $\left(i, \omega^{i}\right)$.
} 
the equality follows from the third equality in (4) and the fact that we took $b=\left(i, \omega^{0}\right)$. So, $p_{a b}(i, \omega)=$ $\frac{1}{c} p_{a b}(i, c \omega)$. Then, by 9 , we have $\phi(i, \omega)=p_{a b}(i, \omega)=\frac{1}{c} p_{a b}(i, c \omega)$, and thus $f_{i}(\omega)=\phi(i, \omega)=$ $\frac{1}{c} p_{a b}(i, c \omega)=\frac{1}{c} \phi(i, c \omega)=\frac{1}{c} f_{i}(c \omega)$. So, scale invariance is also satisfied in this case.

To prove that the preference relation can be represented by an additive power measure, consider any $\omega, \omega^{\prime} \in \mathcal{W D}^{N}$. Note that for every $i \in N$, neutrality to ordinary risk implies that $\left(i, \frac{1}{2} \omega+\frac{1}{2} \omega^{\prime}\right) \sim$ $\left[\frac{1}{2}(i, \omega) ; \frac{1}{2}\left(i, \omega^{\prime}\right)\right]$ and thus $\phi\left(i, \frac{1}{2} \omega+\frac{1}{2} \omega^{\prime}\right)=\phi\left(\left[\frac{1}{2}(i, \omega) ; \frac{1}{2}\left(i, \omega^{\prime}\right)\right]\right)=\frac{1}{2} \phi(i, \omega)+\frac{1}{2} \phi\left(i, \omega^{\prime}\right)$, where the first equality follows from neutrality to ordinary risk and the second from 5 . But then $f_{i}\left(\omega+\omega^{\prime}\right)=f_{i}\left(2\left(\frac{1}{2} \omega+\right.\right.$ $\left.\left.\frac{1}{2} \omega^{\prime}\right)\right)=2 f_{i}\left(\frac{1}{2} \omega+\frac{1}{2} \omega^{\prime}\right)=2 \phi\left(i, \frac{1}{2} \omega+\frac{1}{2} \omega^{\prime}\right)=2\left(\frac{1}{2} \phi(i, \omega)+\frac{1}{2} \phi\left(i, \omega^{\prime}\right)\right)=\phi(i, \omega)+\phi\left(i, \omega^{\prime}\right)=f_{i}(\omega)+f_{i}\left(\omega^{\prime}\right)$, where the second equality follows from scale invariance of $f$. So, additivity is satisfied.

\section{Proof of Theorem 1}

To prove the 'only if' part, note that it follows from Lemma 1 and Proposition 1 that, if expected utility function $\phi$ represents a regular preference relation that satisfies Axioms 5 , 8 , then these preferences can be represented by a utility function $\phi(i, \omega)=\alpha \cdot$ out $_{i}(\omega)+\beta \cdot i n_{i}(\omega)$ for some $\alpha, \beta \in \mathbf{R}$. Then $\phi_{i}\left(\omega^{0}\right)=0$ for all $i \in N$. By Axiom 7, it must hold that $\phi\left(i, \omega^{i}\right)=\alpha(|N|-1)>0=\phi\left(i, \omega^{0}\right)$, and thus $\alpha>0$. By Axiom 6, it must hold that $\phi\left(j, \omega^{i}\right)=\beta \geq 0=\phi\left(j, \omega^{0}\right)$ for all $j \in N \backslash\{i\}$, and thus $\beta \geq 0$. By scale invariance, we can rescale such that $\alpha+\beta=1$, and thus the preferences can be represented by a utility function $\phi(i, \omega)=\alpha \cdot$ out $_{i}(\omega)+(1-\alpha) \cdot \operatorname{in}_{i}(\omega)$ for some $\alpha \in(0,1]$.

To prove the 'if' part, let $\geq$ be the preference relation based on $\phi(i, \omega)=\alpha \cdot \operatorname{out}_{i}(\omega)+(1-\alpha) \cdot \operatorname{in}_{i}(\omega)$ with $\alpha \in(0,1]$, i.e. $(i, \omega) \geq\left(j, \omega^{\prime}\right)$ if and only if $\alpha \cdot$ out $_{i}(\omega)+(1-\alpha) \cdot \operatorname{in}_{i}(\omega) \geq \alpha \cdot \operatorname{out}_{i}\left(\omega^{\prime}\right)+(1-\alpha) \cdot \operatorname{in}_{i}\left(\omega^{\prime}\right)$. It is straightforward to check that $\geq$ satisfies Axiom 5 . Axiom 6 follows since (i) $\alpha \cdot$ out $_{i}(\omega)+(1-\alpha) \cdot \operatorname{in}_{i}(\omega) \geq 0$ for all $\omega \in \mathcal{W} \mathcal{D}^{N}$, and (ii) $\alpha \cdot$ out $_{i}\left(\omega^{\prime}\right)+(1-\alpha) \cdot i n_{i}\left(\omega^{\prime}\right)=0$ for all $\omega^{\prime} \in \mathcal{W} \mathcal{D}_{i}^{N}$. Axiom 7 follows since $\alpha \cdot \operatorname{out}_{i}\left(\omega^{i}\right)+(1-\alpha) \cdot \operatorname{in}_{i}\left(\omega^{i}\right)>0$. Finally, to prove neutrality to ordinary risk, consider $\omega, \omega^{\prime} \in \mathcal{W D}^{N}$ and $i \in N$. Then, for $p \in[0,1]$ we have $\phi\left(i, p \omega+(1-p) \omega^{\prime}\right)=\alpha \cdot$ out $_{i}\left(p \omega+(1-p) \omega^{\prime}\right)+(1-\alpha)$. $\operatorname{in}_{i}\left(p \omega+(1-p) \omega^{\prime}\right)=\alpha \cdot \operatorname{out}_{i}(p \omega)+\alpha \cdot \operatorname{out}_{i}\left((1-p) \omega^{\prime}\right)+(1-\alpha) \cdot \operatorname{in}_{i}(p \omega)+(1-\alpha) \cdot \operatorname{in}_{i}\left((1-p) \omega^{\prime}\right)=$ $p \alpha \cdot$ out $_{i}(\omega)+(1-p) \alpha \cdot$ out $_{i}\left(\omega^{\prime}\right)+p(1-\alpha) \cdot$ in $_{i}(\omega)+(1-p)(1-\alpha) \cdot \operatorname{in}_{i}\left(\omega^{\prime}\right)=p\left(\alpha \cdot\right.$ out $_{i}(\omega)+(1-\alpha) \cdot$ $\left.\operatorname{in}_{i}(\omega)\right)+(1-p)\left(\alpha \cdot \operatorname{out}_{i}\left(\omega^{\prime}\right)+(1-\alpha) \cdot i n_{i}\left(\omega^{\prime}\right)\right)=p \phi(i, \omega)+(1-p) \phi\left(i, \omega^{\prime}\right)=\phi\left(\left[p(i, \omega) ;(1-p)\left(i, \omega^{\prime}\right)\right]\right)$, where the last equality follows from (5). 


\section{Appendix C: Logical independence of the axioms in Theorem 1}

In this appendix, we give four regular utility functions representing preference relations over graph positions that each satisfy three, but not all four, of the additional axioms in Theorem 1 .

1. Consider weights $\left(\alpha_{i}\right)_{i \in N}$ such that $\alpha_{i} \in\left(\frac{1}{2}, 1\right]$ for all $i \in N$, with $\alpha_{i} \neq \alpha_{j}$ for at least one pair of nodes, and consider the utility function over graph positions $\phi(i, \omega)=\alpha_{i}$ out $_{i}(\omega)+\left(1-\alpha_{i}\right) \operatorname{in}_{i}(\omega)$ for all $(i, \omega) \in N \times \mathcal{W} \mathcal{D}^{N}$. The associated preference relation satisfies isolated is the worst, center of the star is strictly better than being isolated, and neutrality to ordinary risk. It does not satisfy anonymity.

2. Consider the utility function over graph positions $\phi(i, \omega)=\operatorname{out}_{i}(\omega)-i n_{i}(\omega)$ for all $(i, \omega) \in N \times \mathcal{W} \mathcal{D}^{N}$. This is known as the Copeland score ([15]). The associated preference relation satisfies anonymity, center of the star is strictly better than being isolated, and neutrality to ordinary risk. It does not satisfy isolated is the worst.

3. Consider the utility function over graph positions $\phi(i, \omega)=1$ for all $(i, \omega) \in N \times \mathcal{W D}^{N}$. The associated preference relation satisfies anonymity, isolated is the worst, and neutrality to ordinary risk. It does not satisfy center of the star is strictly better than being isolated.

4. Consider the utility function over graph positions $\phi(i, \omega)=1$ if $i$ is the center of (a multiple of) a star, i.e., $\omega(i, j)>0$ for all $j \in N \backslash\{i\}$ and $\omega(j, k)=0$ for all $j \in N \backslash\{i\}$ and $k \in N \backslash\{j\}$, and $\phi(i, \omega)=0$ otherwise. The associated preference relation satisfies anonymity, isolated is the worst, and center of the star is strictly better than being isolated. It does not satisfy neutrality to ordinary risk.

\section{References}

[1] Ambec, S., \& Ehlers, L. (2008). Sharing a river among satiable agents. Games and Economic Behavior, 64, 35-50.

[2] Banzhaf, J. (1965). Weighted voting doesn't work: a mathematical analysis. Rutgers Law Review, $19,317-343$.

[3] Bavelas, A. (1948). A mathematical model for group structures. Human Organization, 7, 16-30.

[4] Bavelas, A. (1950). Communication patterns in task-oriented groups. Journal of the Acoustical Society of America, 22, 725-730.

[5] Beauchamp, M. A. (1965). An improved index of centrality. Behavioral Science, 10, 161-163. 
[6] Bergantiños, G., \& Moreno-Ternero, J. D. (2020). Sharing the revenues from broadcasting sport events. Management Science, 66(6), 2417-2431.

[7] Bloch, F., Jackson, M. O., \& Tebaldi, P. (2016). Centrality measures in networks. Working Paper.

[8] Bonacich, P. B. (1972). Factoring and weighting approaches to status scores and clique identification. Journal of Mathematical Sociology, 2, 113-120.

[9] Bonacich, P. B. (1987). Power and centrality: A family of measures. American Journal of Sociology, 92, 1170-1182.

[10] Borgatti, S. P. (2005). Centrality and network flow. Social Networks, 27(1), 55-71.

[11] Bouyssou, D. (1992). Ranking methods based on valued preference relations: A characterization of the net flow method. European Journal of Operational Research, 60, 61-67.

[12] Bouyssou, D., \& Marchant, T. (2018). The $\beta$-ranking and the $\beta$-measure for directed networks: Axiomatic characterizations. Social Networks, 52(1), 145-153.

[13] Bouyssou, D., \& Perny, P. (1992). Ranking methods for valued preference relations: A characterization of a method based on leaving and entering flows. European Journal of Operational Research, 61, 186-194.

[14] Brown, D., \& Housman, D. (1988). Cooperative games on weighted graphs. Internal Report, Worcester Polytechnic Institute, Worcester, MA.

[15] Copeland, A. H. (1951). A Reasonable Social Welfare Function. Mimeographed Notes, University of Michigan Seminar on Applications of Mathematics to the Social Sciences.

[16] de Clippel, G., \& Serrano, R. (2008). Marginal contributions and externalities in the value. Econometrica, 76, 1413-1436.

[17] Demange, G. (2014). A ranking method based on handicaps. Theoretical Economics, 9(3), 915942.

[18] Demange, G. (2017). Mutual rankings. Mathematical Social Sciences, 90, 35-42.

[19] Deng, X., \& Papadimitriou, C. H. (1994). On the complexity of cooperative solution concepts. Mathematics of Operations Research, 19(2), 257-266.

[20] Dequiedt, V., \& Zenou, Y. (2017). Local and consistent centrality measures in parameterized networks. Mathematical Social Sciences, 88, 28-36. 
[21] Du, Y., Lehrer, E., \& Pauzner, A. (2015). Competitive economy as a ranking device over networks. Games and Economic Behavior, 91, 1-13.

[22] Freeman, L. (1977). A set of measures of centrality based on betweenness. Sociometry, 40, 35-41.

[23] Freeman, L. C. (1979). Centrality in social networks: Conceptual clarification. Social Networks, 1, 215-239.

[24] Garg, M. (2009). Foundations of centrality in networks. Mimeo.

[25] Gómez, D., González-Arangüena, E., Manuel, C., Owen, G., del Pozo, M., \& Tejada, J. (2003). Centrality and power in social networks: a game theoretic approach. Mathematical Social Sciences, $46,27-54$.

[26] Goyal, S. (2007). Connections: An Introduction to the Economics of Networks. Princeton University Press.

[27] Harsanyi, J. C. (1959). A bargaining model for the cooperative n-person game. In A. W. Tucker, \& R. D. Luce (Eds.), Contributions to the Theory of Games 4 (pp. 325-335). Princeton, N.J.: Princeton University Press.

[28] Henriet, D. (1985). The Copeland choice function - an axiomatic characterization. Social Choice and Welfare, 2, 49-63.

[29] Herstein, I. N., \& Milnor, J. (1953). An axiomatic approach to measurable utility. Econometrica, 21, 291-297.

[30] Jackson, M. O. (2008). Social and Economic Networks. Princeton University Press.

[31] Katz, L. (1953). A new status index derived from sociometric analysis. Psychometrika, 18, 39-43.

[32] Kitti, M. (2016). Axioms for centrality scoring with principal eigenvectors. Social Choice and Welfare, 46(3), 639-653.

[33] Maniquet, F. (2003). A characterization of the Shapley value in queueing models. Journal of Economic Theory, 109(1), 90-103.

[34] Moulin, H. (1987). Equal or proportional division of a surplus, and other methods. International Journal of Game Theory, 16, 161-186.

[35] Neuefeind, W., \& Trockel, W. (1995). Continuous linear representability of binary relations. Economic Theory, 6, 351-356. 
[36] Newman, M. E. J. (2010). Networks: An Introduction. Oxford University Press.

[37] Nieminen, J. (1974). On centrality in a graph. Scandinavian Journal of Psychology, 15, 322-336.

[38] Nowak, A. S., \& Radzik, T. (1994). The Shapley value for n-person games in generalized characteristic function form. Games and Economic Behavior, 6, 150-161.

[39] Palacios-Huerta, I., \& Volij, O. (2004). The measurement of intellectual influence. Econometrica, 72, 963-977.

[40] Pintér, M. (2014). On the Axiomatizations of the Shapley Value. Habilitation Thesis, Corvinus University of Budapest. Url: http://d85677ad-a-62cb3a1a-s-sites.googlegroups.com/ site/miklospinter/home/kutatas/Habilitacio2014.pdf.

[41] Roth, A. E. (1977). Bargaining ability, the utility of playing a game, and models of coalition formation. Journal of Mathematical Psychology, 16, 153-160.

[42] Roth, A. E. (1977). The Shapley value as a von Neumann-Morgenstern utility. Econometrica, 45(3), 657-664.

[43] Roth, A. E. (1977). Utility functions for simple games. Journal of Economic Theory, 16, 481-489.

[44] Roth, A. E. (1988). The expected utility of playing a game. In A. E. Roth (Ed.), The Shapley Value: Essays in Honor of Lloyd S. Shapley (pp. 51-70). Cambridge University Press.

[45] Rubinstein, A. (1980). Ranking the participants in a tournament. SIAM Journal of Applied Mathematics, 38(1), 108-111.

[46] Sabidussi, G. (1966). The centrality index of a graph. Psychometrika, 31, 581-603.

[47] Sánchez, E., \& Bergantiños, G. (1997). On values for generalized characteristic functions. $O$. $R$. Spektrum, 19, 229-234.

[48] Schmeidler, D. (1969). The nucleolus of a characteristic function game. SIAM Journal of Applied Mathematics, 17, 1163-1170.

[49] Shapley, L. S. (1953). A value for n-person games. In H. W. Kuhn, \& A. W. Tucker (Eds.), Annals of Mathematics Studies 28 (pp. 307-317). Princeton University Press.

[50] Shaw, M. E. (1954). Group structure and the behaviour of individuals in small groups. Journal of Psychology, 38, 139-149. 
[51] Slutzki, G., \& Volij, O. (2006). Scoring of web pages and tournaments - axiomatizations. Social Choice and Welfare, 26, 75-92.

[52] Tijs, S. (1981). Bounds for the core of a game and the $\tau$-value. In O. Moeschlin, \& D. Pallaschke (Eds.), Game Theory and Mathematical Economics (pp. 123-132). North-Holland Publ. Comp., Amsterdam.

[53] Trockel, W. (1989). Classification of budget-invariant monotonic preferences. Economics Letters, $30,7-10$.

[54] Trockel, W. (1992). An alternative proof for the linear utility representation theorem. Economic Theory, 2, 298-302.

[55] van den Brink, R., \& Gilles, R. P. (2000). Measuring domination in directed networks. Social Networks, 22, 141-157.

[56] van den Brink, R., \& Gilles, R. P. (2003). Ranking by outdegree for directed graphs. Discrete Mathematics, 271, 261-270.

[57] van den Brink, R., \& Gilles, R. P. (2009). The outflow ranking method for weighted directed graphs. European Journal of Operational Research, 193, 484-491.

[58] van den Brink, R., \& Pintér, M. (2015). On axiomatizations of the Shapley value for assignment games. Journal of Mathematical Economics, 60, 110-114.

[59] van den Brink, R., \& Rusinowska, A. (2021). The degree ratio ranking method for directed graphs. European Journal of Operational Research, 288(2), 563-575.

[60] van den Nouweland, A., Borm, P., van Golstein Brouwers, W., Bruinderink, R. G., \& Tijs, S. (1996). A game theoretic approach to problems in telecommunication. Management Science, 42(2), 294-303.

[61] von Neumann, J., \& Morgenstern, O. (1944). Theory of Games and Economic Behavior. Princeton: Princeton University Press. 